\title{
AN APPLICATION OF PRIMARY PTD APPROXIMATION: BACKSCATTERING AT TRIANGULAR CYLINDER
}

\author{
Feray HACIVELIOGLU*, Mathematical Department, Gebze Technical University, Turkey, ferayhvo@gmail.com \\ (iD) https://orcid.org/ 0000-0003-0394-3736)
}

Received: 13.09.2020, Accepted: 23.02.2021

Research Article

${ }^{*}$ Corresponding author

DOI: $10.22531 /$ muglajsci.794422

\begin{abstract}
The purpose of the paper is to investigate the backscattering at a triangular cylinder with one soft (electric) face and two hard (magnetic) faces and to reveal the scattering properties of the geometry. In the paper, the backscattered field in the far-zone is introduced using the Physical Theory of Diffraction (PTD), one of the powerful high-frequency approximation techniques. First-order PTD approximation is obtained as a sum of the primary edge waves. Graphical results that have minimums in the radar cross-section represent the importance of geometry in applications of stealth technology. The method used in the paper allows the radar cross section (RCS) to calculate easily using simple trigonometric functions.
\end{abstract}

Keywords: Backscattering, high-frequency approximation, physical optics, physical theory of diffraction, stealth technology, triangular cylinder.

\section{PTD YAKLAŞIMININ BİR UYGULAMASI: ÜÇGENSEL SİLİNDİRDEN GERİ SAÇILIM}

Özet

Bu makalenin amacl, bir yüzeyi soft (elektrik), diğer iki yüzeyi hard (manyetik) olan üçgensel sonsuz silindirden geri saçılımın incelenmesi ve bu geometrinin saçılım özelliklerinin açığa çıkarılmasıdır. Bu makalede, uzak alandaki geri saçılma, etkili yüksek frekans yaklaşımlarından biri olan Kırınımın Fiziksel Teorisi (KFT) ile incelenmiştir. Birinci mertebeden KFT yaklaşımı, birincil ayrıt dalgalarının toplamı şeklinde elde edilmiştir. Radar kesit alanında minimumlara sahip olan grafiksel sonuçlar, bu geometrinin görünmezlik teknolojisinin uygulamalarında önemli olabileceğini göstermektedir. Makalede kullanılan KFT yöntemi, radar kesit alanının (RKA) trigonometrik fonksiyonları kullanarak kolayca hesaplanmasını sağlamaktadır.

Anahtar Kelimeler: Geri saçılım, yüksek frekans yaklaşımı, fizik optik, kırınımın fiziksel teorisi, görünmezlik teknolojisi, üçgensel silindir.

Cite

Hacivelioglu, F., (2021). "An Application of Primary PTD Approximation: Backscattering at Triangular Cylinder", Mugla Journal of Science and Technology, 7(1), 1-6.

\section{Introduction}

One of the basic techniques used for reduction of radar cross section (RCS) playing key role in stealth technology is shaping. Polygonal cross section cylinders are useful in application of stealth technology due to their properties of reducing RCS [1]. Bi-static and monostatic scattering by cylinders with triangular cross section are studied in many papers using different analytical and numerical techniques. Diffraction by infinite polygonal cylinders with both Dirichlet and Neumann boundary conditions is investigated using Geometrical theory of diffraction (GTD) in [2]. Higher order diffraction by conducting triangular cylinder using Uniform theory of diffraction is studied and showed the correctness of the results by comparing them Method of Moments (MoM) results in [3] where comparison of first-order diffraction and higher-order diffraction is absent. The results obtained from backscattering by triangular cylinder and hexahedron studied by modified equivalent current method is compared with experimental results and got excellent agreement in [4]. In [5] electromagnetic backscattering from dielectric cylinder whose base is isosceles triangle is studied using analytical regularization method. Bi-static and monostatic scattering by totally soft and totally hard equilateral triangular cylinder are investigated in [6] by using Physical Optics (PO) and Physical Theory of Diffraction (PTD). Sukharevsky et al. investigate the interaction between Geometric Optics (GO)-like ray effects 
responsible for enhanced reflection from right angle prism incase base illumination and resonances responsible for the peaks in the total field and absorption cross-section at the natural-mode frequency in a dielectric cylinder of triangular cross-section with rounding edges using Muller boundary integral equation in [7]. They show that interplay of ray effects and resonance is complicated and when the electric size of the scatterer becomes larger, both effects become more notable. They also demonstrate that by changing prism parameters and orientation carefully RCS can be controlled. Sukharevsky et al. indicate that although dielectric triangular cylinders can be helpful in application of stealth technology, researchers do not put emphasis on scattering from such geometries. Firstorder bi-static PTD approximation of triangular cylinders with combination of soft and hard faces is investigated in [8], and the obtained results are validated by the MoM results. Backscattering at triangular cylinder with two soft faces and one hard face is investigated in [9]. Lately, in [10] physical theory of diffraction for triangular cylinders with faces which are the combination of soft (electric) and hard (magnetic) sections is studied. In [10] authors show that the specular reflection can be decreased significantly and shifted to other directions by using correct combinations of the soft and hard sections on the faces of the triangular cylinder.

In the present paper, a high-frequency approximation for the diffracted field by a triangular cylinder with two hard (magnetic) faces and one soft (electric) face in terms of PO and PTD is introduced. Section 2 presents the review of the canonical wedge problem for the sake of the completeness and illustrates the geometry of the problem and the directivity functions of the edge waves for the backscattering PO and PTD fields. Radar cross section is demonstrated graphically for different angular parameters in Section 3. Finally, conclusions are presented in Section 4.

\section{Backscattering at Triangular Cylinder}

We will first review the wedge problem for the sake of the completeness. We need the asymptotic solutions of the canonical wedges problem with 1) hard (magnetic) faces, and 2) one face is soft (electric) and the other is hard (magnetic) for the problem of this paper. Ufimtsev investigated the wedge problem with 1) totally hard (magnetic) faces, 2) one face is soft (electric) and the other is hard (magnetic) using PTD in [6] and [11], respectively. He obtained the exact solutions of the Helmholtz equation under the 1) hard boundary conditions (BCs), 2) soft-hard BCs in [6] and [11], respectively and then he converted the series in the exact solutions to the Sommerfeld-type integral equations for the asymptotic evaluation. He found that the diffracted fields can be represented asymptotically as,

$$
u^{d i f}=f\left(\phi, \phi_{0}\right) \frac{e^{i(k r+\pi / 4)}}{\sqrt{2 \pi k r}}
$$

where $f\left(\phi, \phi_{0}\right)=f_{h}\left(\phi, \phi_{0}\right)$, directivity pattern of the edge waves for totally hard wedge, with

$$
\begin{aligned}
f_{h}\left(\phi, \phi_{0}\right) & =\frac{1}{n} \sin \left(\frac{\pi}{n}\right)\left(\frac{1}{\cos (\pi / n)-\cos \left(\left(\phi-\phi_{0}\right) / n\right)}\right. \\
& \left.+\frac{1}{\cos (\pi / n)-\cos \left(\left(\phi+\phi_{0}\right) / n\right)}\right)
\end{aligned}
$$

for the case 1 , and $f\left(\phi, \phi_{0}\right)=f_{s h}\left(\phi, \phi_{0}\right)$, directivity pattern of the edge waves for wedge with one face soft and the other hard, with

$$
\begin{aligned}
& f_{s h}\left(\phi, \phi_{0}\right)=\frac{2}{n} \sin \left(\frac{\pi}{2 n}\right) * \\
& \left(\frac{\cos \left(\left(\phi-\phi_{0}\right) / 2 n\right)}{\cos (\pi / n)-\cos \left(\left(\phi-\phi_{0}\right) / n\right)}-\frac{\cos \left(\left(\phi+\phi_{0}\right) / 2 n\right)}{\cos (\pi / n)-\cos \left(\left(\phi+\phi_{0}\right) / n\right)}\right)
\end{aligned}
$$

for the case 2 . Here, $k=2 \pi / \lambda$ is the wave number, $\lambda$ wavelength. $n=\alpha / \pi, \alpha$ is the exterior angle of the wedge. $\phi_{0}$ and $\phi$ are incident and observation angles, respectively. He also investigated bi-static scattering and backscattering from totally soft and totally hard equilateral triangular cylinder in [6]. In [8] bi-static scattering from isosceles triangular cylinder with combinations of soft (electric) and hard (magnetic) faces is investigated using first-order PTD approximation. The aims of this paper are to investigate the case similar to Case $C$ in [8] deeply for the backscattering case which can be important in low-observability and to compare the results with the results in [9] where the triangular cylinder has two soft faces and one hard face (2soft+1hard).

The geometry of the study is the isosceles triangular cylinder with one soft face and two hard faces (1soft+2hard) depicted in 2D in Fig 1. The points 1, 2, and 3 denote the edges whose Cartesian coordinates are $(0,0),(h, a)$, and $(h,-a)$, respectively. Here $h=l \cos \gamma$, $a=l \sin \gamma . l$ is the length of the faces 1-2 and 1-3. Faces 1-3 and 2-3 are imposed to hard boundary condition and face 1-2 is imposed soft boundary condition.

Incident wave is given by

$u^{i n c}=\exp \left(-i k\left(x \cos \varphi_{0}+y \sin \varphi_{0}\right)\right), \quad 0 \leq \varphi_{0} \leq 2 \pi$

Time dependence $\exp (-i \omega t)$ is suppressed throughout the paper.

Scattered field is investigated in the direction $\varphi=\varphi_{0}$. 


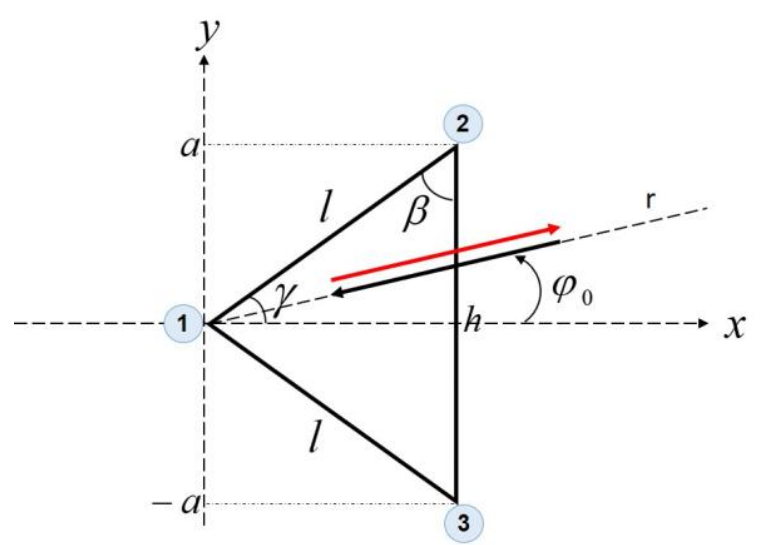

Figure 1. 2D geometry of the problem.

Far field ( $r \sqcup k l^{2}$ ) can be calculated as the sum of the three edge waves

$u^{d i f}=\sum_{m=1}^{3} \varepsilon_{m} u_{0}(m) f\left(\phi_{m}\right) \frac{e^{i\left(k r_{m}+\pi / 4\right)}}{\sqrt{2 \pi k r_{m}}}$

Here $\left(r_{m}, \phi_{m}\right), \quad m=1,2,3$ are local polar coordinates where local angles are measured from the illuminated face of the cylinder. $r_{1,2,3}$ are measured from the edges $1,2,3$ and if we let $r_{1}=r$, then $r_{2}=r-h \cos \varphi-a \sin \varphi$ and $r_{3}=r-h \cos \varphi+a \sin \varphi$ for the far field where $r \square k l^{2}$ (Fig. 2 (top)) where $\varphi$ is measured from positive x-axis. $u^{\text {dif }}$ in (5) represents $\mathrm{PO}$ and PTD field depending on the function $f$. If it is PO (PTD) diffracted field, $f$ is $f_{s}^{(0)}$ and $f_{h}^{(0)}\left(f_{s}, f_{h}\right.$ and $\left.f_{s h}\right) . u_{0}(1)=u_{0}, u_{0}(2)=u_{0} e^{i k(h \cos \varphi+a \sin \varphi)}$ and $u_{0}(3)=u_{0} e^{i k(h \cos \varphi-a \sin \varphi)}$. Hence the diffracted field in (5) will be

$u^{d i f}=u_{0} \sum_{m=1}^{3} \Phi_{m}(\varphi) \frac{e^{i(k r+\pi / 4)}}{\sqrt{2 \pi k r}}$

where $\Phi_{m}(\varphi)=\varepsilon_{m} f(m) e^{i \psi_{m}}$ with $f(m)=f\left(\phi_{m}\right)$ and the quantities $\psi_{1,2,3}$ are defined as $\psi_{1}=0$, $\psi_{2}=-2 k l \cos (\gamma-\varphi) \quad$ and $\quad \psi_{3}=-2 k l \cos (\gamma+\varphi) . \quad \varepsilon_{m}=1$ when the edge $m$ is seen, otherwise $\varepsilon_{m}=0$.
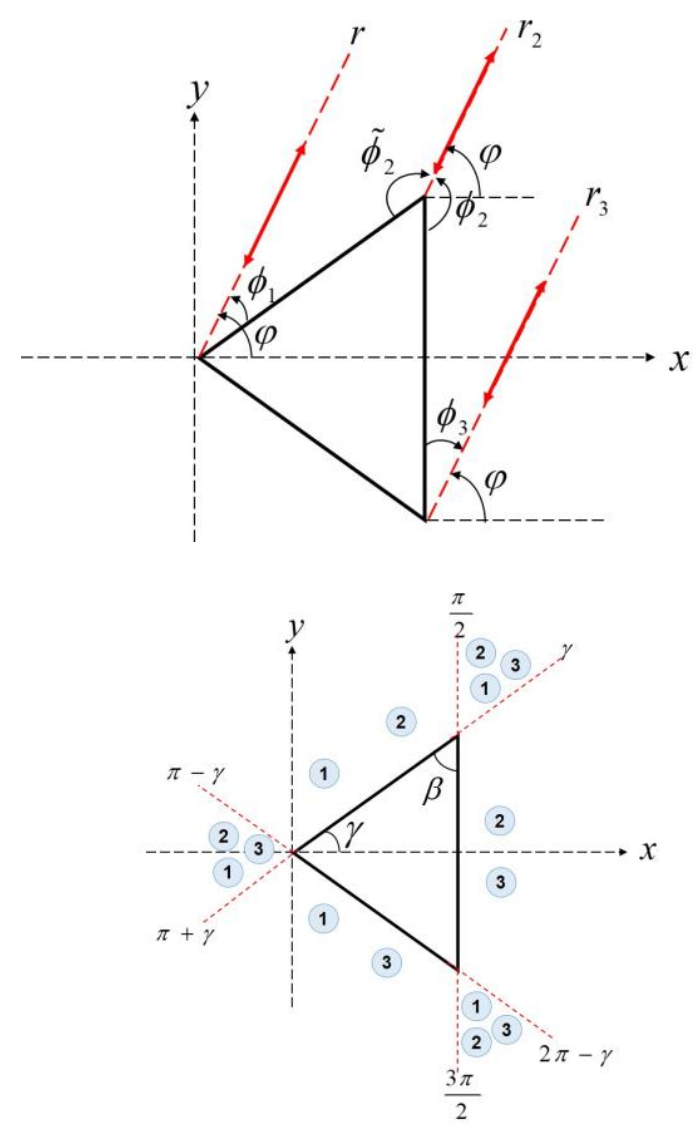

Figure 2. Local polar coordinates (top), seen edges in regions (bottom)

Fig. 2 (bottom) depicts that in which region which edges are seen.

Then we can define the parameters $\varepsilon_{1,2,3}$ as follows

$\varepsilon_{1}=\left\{\begin{array}{ll}1, & \text { otherwise } \\ 0, & -\gamma \leq \varphi \leq \gamma\end{array} \quad \varepsilon_{2}=\left\{\begin{array}{cc}1, & \text { otherwise } \\ 0, & \pi+\gamma \leq \varphi \leq 3 \pi / 2\end{array}\right.\right.$, $\varepsilon_{3}=\left\{\begin{array}{lc}1, & \text { otherwise } \\ 0, & \pi / 2 \leq \varphi \leq \pi-\gamma\end{array}\right.$

If we use local polar coordinates in (2) and (3), we have $\begin{aligned} f_{s h}(1) & =\frac{2}{n_{1}} \sin \frac{\pi}{2 n_{1}}\left(\frac{1}{\cos \left(\pi / n_{1}\right)-1}, \quad \gamma \leq \varphi \leq 2 \pi-\gamma\right. \\ & \left.+\frac{\cos \left((\varphi-\gamma) / n_{1}\right)}{\cos \left(\pi / n_{1}\right)-\cos \left((2 \varphi-2 \gamma) / n_{1}\right)}\right)\end{aligned}$

$$
\begin{aligned}
f_{\text {sh }}(2) & =\frac{2}{n_{2}} \sin \frac{\pi}{2 n_{2}}\left(\frac{1}{\cos \left(\pi / n_{2}\right)-1}\right. \\
& \left.+\frac{\cos \left((\pi+2 \varphi) / 2 n_{2}\right)}{\cos \left(\pi / n_{2}\right)-\cos \left((\pi+2 \varphi) / n_{2}\right)}\right)
\end{aligned}
$$




$$
\begin{aligned}
f_{s h}(2) & =\frac{2}{n_{2}} \sin \frac{\pi}{2 n_{2}}\left(\frac{1}{\cos \left(\pi / n_{2}\right)-1} \quad, 3 \pi / 2 \leq \varphi \leq 2 \pi\right. \\
& \left.-\frac{\cos \left((\varphi+\gamma) / n_{2}\right)}{\cos \left(\pi / n_{2}\right)-\cos \left((2 \varphi+2 \gamma) / n_{2}\right)}\right)
\end{aligned}
$$$$
f_{h}(3)=\frac{1}{n_{3}} \sin \frac{\pi}{n_{3}}\left(\frac{1}{\cos \left(\pi / n_{3}\right)-1}\right.
$$$$
\left.+\frac{1}{\cos \left(\pi / n_{3}\right)-\cos \left((\pi-2 \varphi) / n_{3}\right)}\right)
$$$$
0 \leq \varphi \leq \pi / 2
$$

$$
\begin{aligned}
f_{h}(3) & =\frac{1}{n_{3}} \sin \frac{\pi}{n_{3}}\left(\frac{1}{\cos \left(\pi / n_{3}\right)-1}\right. \\
& \left.+\frac{1}{\cos \left(\pi / n_{3}\right)-\cos \left((2 \pi-2 \varphi-2 \gamma) / n_{3}\right)}\right) \\
\pi-\gamma & \leq \varphi \leq 2 \pi
\end{aligned}
$$

Here, $n_{1,2,3}$ are the edge parameters equal to $n_{1}=\frac{2 \pi-2 \gamma}{\pi}$ and $n_{2}=n_{3}=\frac{2 \pi-\beta}{\pi}$.

The functions $f_{s h}(2)$ and $f_{h}(3)$ have singularities in the direction $\varphi=0^{0}$. However, in the directivity function

$$
\Phi^{P T D}(\varphi)=f_{s h}(2) e^{i \psi_{2}}+f_{h}(3) e^{i \psi_{3}},
$$

these singularities cancel each other and (6) is finite and its value is

$$
\begin{aligned}
& \Phi^{P T D}(0)=-i 2 k l \sin \gamma e^{-i 2 k l \cos \gamma} \\
& -\frac{1}{n_{2}}\left(\cot \left(\frac{\pi}{n_{2}}\right)+\frac{1}{2} \tan \left(\frac{\pi}{2 n_{2}}\right)+\frac{\sin \left(\frac{\pi}{n_{2}}\right)}{\cos \left(\frac{\pi}{n_{2}}\right)-1}\right) \mathrm{e}^{-i 2 k l \cos \gamma}
\end{aligned}
$$

In addition, in this direction, $\mathrm{PO}$ directivity function is $\Phi^{P O}(0)=-i 2 k l \sin \gamma e^{-i 2 k l \cos \gamma}$

which is the first term of the (13). It is evaluated using (6) with (3.53) in [6]. Second term on the right hand side of (13) is fringe fields produced by the edges 2 and 3. Fringe field is the field caused by the diffraction from the sharp edges of the cylinder and it is defined as the difference between PTD and PO:

$$
\text { Fringe=PTD-PO. }
$$

\section{Numerical Results}

Using above equations we calculate the normalized backscattering cross-section [6]

$$
\sigma_{\text {norm }}=|\Phi(\varphi) / k l|^{2}
$$

for the triangular cylinder with one soft face and two hard faces (1soft+2hard cylinder). Here, $\varphi$ is the incident and observation angle which is measured from the positive $\mathrm{x}$-axis. The graphics are plotted on the decibel scale for the parameter $\gamma=\pi / 6$ and $k l=6 \pi$ when $l=3 \lambda$ which satisfies the application of asymptotic theory. In Fig.7 and Fig. 8, $\gamma$ varies although the other parameters keep same.

In Fig. 3, PTD and PO fields are compared. PTD and PO fields in Fig. 3 were calculated according to the (6) with (7)-(11) and with (3.53) in [6], respectively. It is observed that, in the regions where all three edges are seen, PTD and PO fields do not agree well due to the fringe fields coming from the all edges. It is also observed that PO backscattering field has minimums caused by the anti-phase contributions from the soft face 1-2 and hard face 1-3. Similar minimums are observed in the PTD field which are shifted due to the asymmetric fringe fields. These minimums reveal the most important property of this geometry: backscattering at triangular cylinder with one soft face and two hard faces can be important in design of low-observable objects.

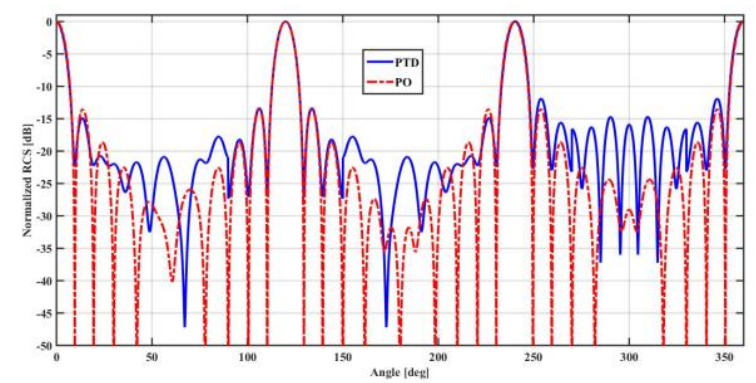

Figure 3. PTD and PO backscattering for 1 soft+2hard cylinder

In Fig. 4 and Fig. 5, backscattered PTD and PO fields for the cylinder with one soft face and two hard faces (the curve 1 soft +2 hard), triangular cylinder with totally soft faces (the curve Totally Soft) and triangular cylinder with totally hard faces (the curve Totally Hard) are compared. Backscattered PTD and PO fields at totally soft and totally hard cylinders were calculated according to the Section 5.2.4 and Section 5.2.2 in [6]. Since PO directivity pattern of totally soft and totally hard cylinders differ only sign in [6], their normalized RCS are the same (Fig.5). However, backscattered PO field at $1 \mathrm{soft}+2$ hard cylinder in the regions $40^{\circ}<\varphi<80^{\circ}$ and $160^{\circ}<\varphi<200^{\circ}$ differ from the backscattered field at totally soft and totally hard cylinders. Because, in these regions where all the three edges are seen, PO field consists of the sum of the fields due to the illumination of both soft and hard faces. In another region where all the edges are seen, $270^{\circ}<\varphi<330^{\circ}$, PO field at the 1 soft +2 hard cylinder is same as the PO field at totally soft and totally hard cylinder since it consists of the sum of the fields caused by the illumination of only hard faces. As expected, in Fig.3-5, the field values at $\varphi=0^{\circ}$ and $\varphi=360^{\circ}$ are the same. 


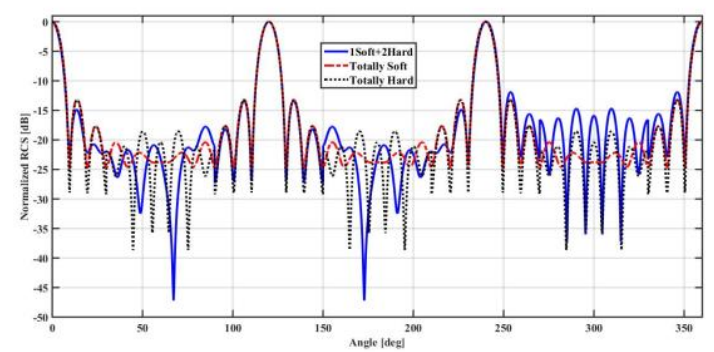

Figure 4. Comparison of the PTD fields for 1 soft+2hard, totally soft and totally hard cylinders

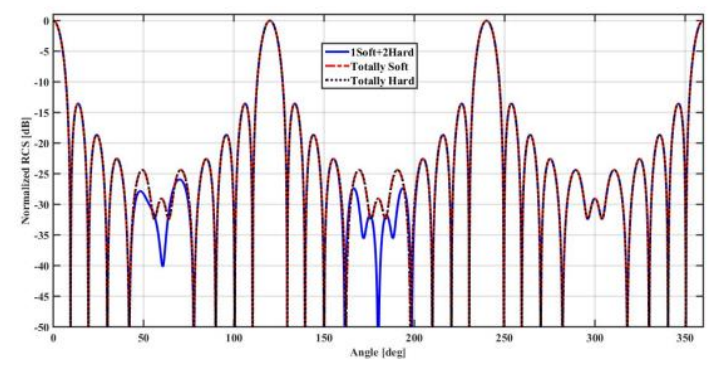

Figure 5. Comparison of the PO fields for 1 soft+2hard, totally soft and totally hard cylinders

In Fig. 3-5, it is seen that scattered field has extra minimum values at $\varphi=180^{\circ}$ which are not seen in the figures of [9]. This minimum is the result of anti-phase contributions from the soft face 1-2 and hard face 1-3. Hence, the triangular cylinder with one soft face and two hard faces has an advantage over the cylinder with two soft faces and one hard face in the sense of lowobservability. The difference of the PTD fields between the $1 \mathrm{soft}+2$ hard triangular cylinder and $2 \mathrm{soft}+1$ hard triangular cylinder in [9] is depicted in Fig. 6.

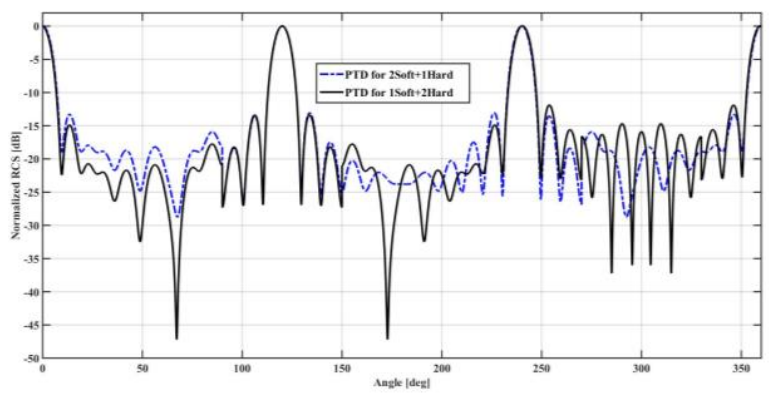

Figure 6. Comparison of the PTD fields for the 1soft +2 hard and 2 soft +1 hard triangular cylinders.

In Fig. 7 and Fig. 8, the effects of the angle $\gamma$ are seen. As expected, since the GO boundaries (incident and reflected boundaries) for the edges are changed when $\gamma$ changes, maximums and minimums of the PO and PTD fields are shifted.

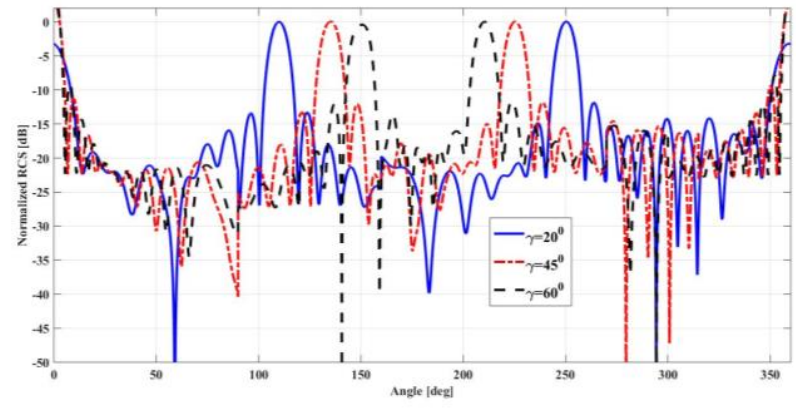

Figure 7. Comparison of the PTD fields for different $\gamma$ values

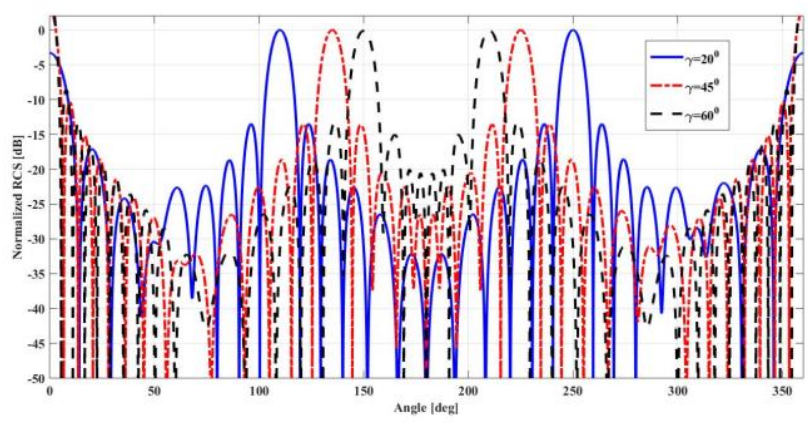

Figure 8. Comparison of the PO fields for different $\gamma$ values

In Fig. 9, PTD and PO fields are plotted for the angle $\gamma=0.5^{\circ}$. When the angle $\gamma$ approaches zero, the triangle cylinder transforms to the soft-hard strip in [12] with a different orientation. Fig. 9 represents that the PO and PTD fields agree well with those of the fields for the softhard strip in Fig. 3 in [12]. The strong asymmetry in the scattered field due to the strong asymmetry on the faces of the triangle cylinder becomes visible as the angle $\gamma$ approaches zero.

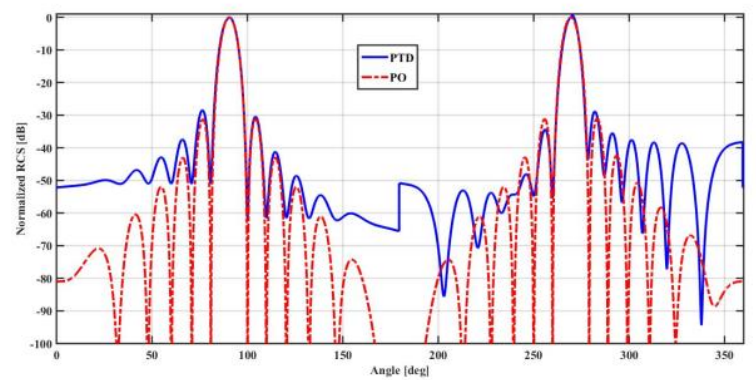

Figure 9. PTD and PO fields for triangular cylinder with $\gamma=0.5^{0}$

\section{Conclusion}

Backscattering at isosceles triangular cylinder with one soft (electric, Dirichlet BC) face and two hard (magnetic, Neumann BCs) faces has been investigated in this paper. First-order asymptotics using physical theory of 
diffraction (PTD) has been derived. The problem of this paper is also studied in [8] for the bi-static case. The results in this paper cannot be obtained directly by substituting $\varphi=\varphi_{0}$ in the results in [8] since the relations between global polar coordinates and local polar coordinates change. For this reason, all the functions of the problem must be derived from the beginning. As a result, derivations in this study do not exist in the literature. In addition, normalized radar cross section (RCS) for the equilateral $\left(\gamma=30^{\circ}\right)$ triangular cylinder with one soft face and two hard faces is compared with the results from the totally soft and totally hard cylinders and with the results from the triangular cylinder with two soft faces and one hard face in [9], numerically. Moreover, the effect of the angle $\gamma$ to the PO and PTD fields is emphasized. It is observed that triangular cylinders with one soft face and two hard faces can be important in design of low-observable objects due to the minimums in the RCS.

\section{References}

[1]. Ufimtsev, P. Ya., "Comments on diffraction principles and limitations for RCS reduction techniques", Proc. IEEE, Vol. 84, No. 12, pp. 1828-1851, 1996.

[2]. Morse, B. J., "Diffraction by polygonal cylinders", Journal of Mathematical Physics, Vol. 5, No. 2, pp. 199-214, 1964.

[3]. Wu, J., and Mostafavi, M., "Higher order UTD analysis of a conducting triangular cylinder excited by a magnetic line source", IEEE Transaction on Magnetics, Vol. 29, No. 2, pp. 1650-1652, 1993.

[4]. Sunahara, Y., Kaniya, S., Aoki, H., Sato, S., Mano, S., "Backscattering from triangular cylinder and hexahedron", Antennas and Propagation Society International Symposium IEEE Philadelphia, USA, 1986.

[5]. Illyashenko, L. N., "Electromagnetic backscattering from a triangular dielectric cylinder", 9th International Conference on Mathematical Methods in Electromagnetic Theory, Kiev, Ukraine, 2002

[6]. Ufimtsev, P. Ya., Fundamentals of the Physical Theory of Diffraction, John Wiley \& Sons, Inc., Hoboken, USA, First ed. 2007, second ed. 2014.

[7]. Sukharevsky, I. O., Nosich, A. I., and Altintas, A., "Manipulation of backscattering from a dielectric cylinder of triangular cross-section using the interplay of GO-like ray effects and resonances", IEEE Transactions on Antennas Propagation, Vol. 63, No. 5, pp. 2162-2168, 2015.

[8]. Hacivelioglu, F., Apaydin, G., Sevgi, L., Ufimtsev, P. Ya., "Diffraction at trilateral cylinders with combinations of soft and hard faces: first-order PTD approximation", Electromagnetics, Vol. 38, No. 4, pp. 217-225, 2018.

[9]. Hacivelioglu, F., "Backscattering from soft-hard triangular cylinder: Primary PTD approximation", TWMS Journal of Applied and Engineering Mathematics, Vol. 10, No. 1, pp. 181-189, 2020.

[10]. Ufimtsev, P. Ya., Apaydin, G., "Cancellation and shifts of specular reflections from soft-hard triangular cylinders", IEEE Transactions on Antennas and Propagation, 19 August 2020.

[11]. Ufimtsev, P. Ya., "Diffraction at a wedge with one face electric and the other face magnetic", IEEE Antennas and Propagation Magazine, Vol. 55, No. 5, pp. 63-73, 2013.

[12]. Hacivelioglu, F., Sevgi, L., and Ufimtsev, P. Ya., "Backscattering from a soft-hard strip: Primary edge waves approximations", IEEE Antennas and Wireless Propagation Letters, Vol. 12, pp. 249-252, 2013. 